\title{
A New Easy Camera Calibration Technique Based on Circular Points
}

\author{
Xiaoqiao Meng, Hua Li and Zhanyi Hu \\ National Laboratory of Pattern Recognition (NLPR) \\ Chinese Academy of Sciences (CAS) \\ P.O.Box 2728, Beijing 100080, P.R.China \\ mengxq@nlpr.ia.ac.cn
}

\begin{abstract}
Inspired by Zhang's work, a new easy technique for calibrating a camera based on circular points is proposed. The proposed technique only requires the camera to observe a newly designed planar calibration pattern (referred to as the model plane hereinafter) which includes a circle and a pencil of lines passing through the circle's center, at a few (at least three) different unknown orientations, then all the five intrinsic parameters can be determined linearly. The main point of our new technique is that it needs to know neither metric measurement on the model plane, nor the correspondences between points on the model plane and image ones, hence it can be done fully automatically. The proposed technique is particularly useful for those people who are not familiar with computer vision. Experiments with simulated data as well as with real images show that our new technique is robust and accurate.
\end{abstract}

\section{Introduction}

Camera calibration is an essential step to extract 3D information from 2D images. An abundant literature on camera calibration has appeared during the last decade. With the increasing popularity of cameras used in offices and families, many people who are not experts in computer vision look more and more concerned with an easy and cheap calibration technique to help them to perform vision tasks.

To this end, Zhang recently proposed a flexible camera calibration technique by replacing an expensive classical calibration grid with a planar pattern [1]. Zhang's technique needs to print a dotted sheet (see Figure1) as the model plane, and the Euclidean coordinates of every dot on the model plane should be measured accurately. After taking a few images of the model plane at different orientations by moving either the model plane or the camera, the homographies between the model plane and its projections can be determined, then camera's intrinsic parameters can be derived linearly from these homographies. Zhang's technique is flexible and cheap, its accuracy is generally higher than self-calibration. Hence it is particularly suitable to desktop 


\section{:3:}

Figure 1: Zhang's planar pattern

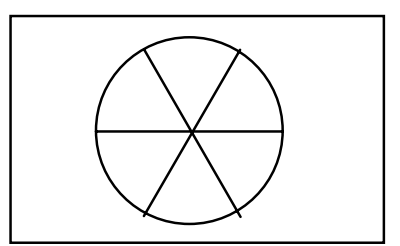

Figure 2: Proposed planar pattern

vision system (DVS) applied in offices and families. However a major drawback in Zhang's technique is that it firstly needs to measure the Euclidean coordinates of every corner on the model plane, and secondly correspondences between points on the model plane and their projections need to be established. The above two requirements are obviously inconvenient to users, in particular to those who are not familiar with computer vision. To overcome this drawback, a novel model plane (see Figure 2) is designed and used in this paper. It is composed of a circle and a pencil of lines passing through the circle's center (referred to as the model circle and the model lines later on). With this new model plane, our calibration technique needs neither physical measurement for the model plane, nor point correspondences, and the whole calibrating process can be done totally automatically.

\section{Theory}

\subsection{Camera model and concept of circular points}

The camera model is assumed to be a pinhole one. The following notation is used in this paper: An image point is denoted by $\mathbf{m}=[u, v]^{T}$, a 3D point is denoted by $\mathrm{M}=[X, Y, Z]^{T}$, and their homogeneous coordinates are denoted by $\tilde{\mathbf{m}}=[u, v, t]^{T}$ and $\tilde{\mathrm{M}}=[X, Y, Z, t]^{T}$ respectively. Then the imaging process from a $3 \mathrm{D}$ point $\mathrm{M}$ to its image $\mathrm{m}$ can be expressed as:

$$
s \tilde{\mathbf{m}}=K\left[\begin{array}{ll}
R & \mathbf{t}
\end{array}\right] \tilde{\mathrm{M}}
$$

Where $\mathrm{s}$ is a non zero scale factor, $\left(\begin{array}{ll}R & \mathbf{t}\end{array}\right)$ are the rotation matrix and translation vector from the world system to the camera system, and $K$ is the camera matrix with the following explicit form:

$$
K=\left[\begin{array}{ccc}
\alpha & \gamma & u_{0} \\
0 & \beta & v_{0} \\
0 & 0 & 1
\end{array}\right]
$$

We will first consider that the world is embedded in a 3D projective space. In this projective space, points satisfying the equation $t=0$ are called points at infinity. They form the plane at infinity which is a $2 \mathrm{D}$ projective subspace embedded in the $3 \mathrm{D}$ projective space. In the plane at infinity, points satisfying $\tilde{\mathrm{M}}^{\mathrm{T}} \tilde{\mathrm{M}}=0$ constitute the 
absolute conic $\omega$. By using (1), we can easily verify that the image of $\omega$ (IAC) is the conic $K^{-T} K^{-1}$. This indicates that IAC encapsulates all the information about camera's intrinsic parameters. Hence if we can determine IAC, we can easily derive all the intrinsic parameters, e.g., via Cholesky factorisation [5].

Without loss of generality, we can assume the model plane lies on the $X-Y$ plane in the world coordinate system, so the equation of the model plane is $Z=0$. Let us denote the ith column of the rotation matrix $R$ by $\mathbf{r}_{i}$, we have:

$$
s\left[\begin{array}{l}
u \\
v \\
1
\end{array}\right]=K\left[\begin{array}{llll}
\mathbf{r}_{1} & \mathbf{r}_{2} & \mathbf{r}_{3} & \mathbf{t}
\end{array}\right]\left[\begin{array}{l}
x \\
y \\
0 \\
t
\end{array}\right]=K\left[\begin{array}{lll}
\mathbf{r}_{1} & \mathbf{r}_{2} & \mathbf{t}
\end{array}\right]\left[\begin{array}{l}
x \\
y \\
t
\end{array}\right]
$$

From (2), a point $[x, y, z, t]^{T}$ on the model plane can also be defined by 2D homogenous coordinates $[x, y, t]^{T}$. According to the projective geometry theory, points on the model plane satisfying the equation $t=0$ form the line at infinity of the model plane (denoted by $l_{\infty}$ ). Now we consider two specific points $I(1, i, 0,0) 、 J(1,-i, 0,0)$ on $l_{\infty}$ (generally called the circular points) [6]. Obviously both $I$ and $J$ satisfy the equation $\tilde{\mathrm{M}}^{\mathrm{T}} \tilde{\mathrm{M}}=0$, so $I$ and $J$ are points on the absolute conic $\omega$. If the images of $I$ and $J$ are denoted by $I_{m}$ and $J_{m}$, then $I_{m}$ and $J_{m}$ should lie on IAC, which yields:

$$
I^{T}{ }_{m} K^{-T} K^{-1} I_{m}=0, \quad J^{T}{ }_{m} K^{-T} K^{-1} J_{m}=0
$$

Since $I$ and $J$ are conjugated points, under the perspective transformation, $I_{m}$ and $J_{m}$ are also conjugated ones. Hence the two equations in (3) are actually identical. However, each equation in (3) can actually produce two linear constraints on IAC by enforcing both its real and imaginary parts to zero. i.e.,

$$
\operatorname{Re}\left(I^{T}{ }_{m} K^{-T} K^{-1} I_{m}\right)=0, \operatorname{Im}\left(I^{T}{ }_{m} K^{-T} K^{-1} I_{m}\right)=0
$$

Now let us look at the newly designed model plane (in Figure 2). As shown in Figure 2, there is a pencil of lines passing through the circle's center $O$. If we assume that the coordinates of $O$ is $\left(O_{x}, O_{y}, 0\right)$ and the circle's radius is $r$ which is unknown, then the circle equation in homogenous coordinates is:

$$
\left(x-O_{x} t\right)^{2}+\left(y-O_{y} t\right)^{2}=r^{2} t^{2}
$$

To compute the intersecting points of the line at infinity $l_{\infty}$ with circle $O$, we combine the equation of $l_{\infty} \quad(t=0)$ and $(4)$ to give:

$$
x^{2}+y^{2}=0 \text {. }
$$

The solution is $y= \pm i x$. That is to say, the two intersecting points can be expressed as $(1, \pm i, 0)$ in homogeneous coordinates, which are independent of $O_{x} 、 O_{y} 、 r$. This means also that "any circle in the model plane should intersects the line at infinity of the model plane at two circular points" [2]. Accordingly in the image plane, the image of the line at infinity should intersect the image of the model circle at the image of the two circular points.

In most cases, the image of the model circle is an ellipse, which can be directly extracted from image. Hence if we can get the image of the line at infinity (generally called the vanishing line), then we can obtain the images of the two circular points. 


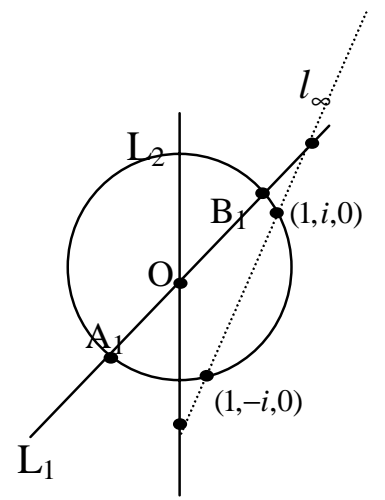

Figure 3: From model plane to image plane

\subsection{Computing the vanishing line}

As sketched in Figure 3, line $L_{1}$ which passes through the model circle's center $O$ intersects the circle at point $A_{1} 、 B_{1}$, and intersects the vanishing line $l_{\infty}$ at $C_{1}$. Based on the theory of projective geometry, the cross-ratio of collinear points $A_{1} 、 B_{1} 、 O 、 C_{1}$ will be -1 , i.e.,

$$
\left(A_{1} B_{1}, O C_{1}\right)=\frac{A_{1} O}{B_{1} O} / \frac{A_{1} C_{1}}{B_{1} C_{1}}=-1
$$

Since $O$ is the midpoint of $A_{1} B_{1}$ and $C_{1}$ is a point at infinity, we can also say that points $A_{1} 、 B_{1}$ harmonically conjugate with respect to points $O 、 C_{1}$. Suppose the corresponding images points of $A_{1} 、 B_{1} 、 O 、 C_{1}$ are $m_{A 1} 、 m_{B 1} 、 m_{O} 、 m_{C 1}$ respectively, since collinearity and cross-ratio are both projective invariant, we may easily conclude that $m_{A 1} 、 m_{B 1} 、 m_{O} 、 m_{C 1}$ are also collinear and their cross-ratio is -1 . Based on these two properties, we can obtain the following two equations:

$$
\begin{aligned}
& \left(m_{A 1} \times m_{B 1}\right) \cdot m_{C 1}=0 \\
& \frac{\left|m_{A 1} m_{O}\right|}{\left|m_{B 1} m_{O}\right|} / \frac{\left|m_{A 1} m_{C 1}\right|}{\left|m_{B 1} m_{C 1}\right|}=-1
\end{aligned}
$$

From the above two equations, $m_{C 1}$ can be computed, and obviously $m_{C 1}$ is on the vanishing line. If there exist more lines on the model plane similar to $L_{1}$, more points on the vanishing line can be similarly computed. Then we can finally obtain the vanishing line by a least squares fitting.

As described above, during computing $m_{C 1}$, we have assumed that points $m_{A 1}$ 、 $m_{B 1} 、 m_{O}$ are collinear. In practice, such a collinearity usually does not hold due to noise and errors introduced in the line detecting process, and extracted image lines $\left\{l_{i} i=1,2, \cdots\right\}$ do not in practice always intersect with each other precisely at the image of the model circle's center. In this case, we define the following cost function to minimize with respect to point $m_{0}$ :

$$
E=\sum_{i} d^{2}\left(m_{O}, l_{i}\right)
$$

Where $d\left(m_{O}, l_{i}\right)$ is the distance from point $m_{O}$ to line $l_{i}$. This is a standard nonlinear minimization problem, which can be solved with Levenberg-Marquardt 
Algorithm or others. After obtaining $m_{O}$, we back-project $m_{O}$ onto every $l_{i}$ to obtain $m_{O i}$. Then when using (5) to compute $m_{C i}$, we will replace $m_{O}$ by $m_{O i}$ in order to ensure $m_{A 1} 、 m_{B 1} 、 m_{O}$ are indeed collinear.

\subsection{Extracting ellipse and lines}

Accurately extracting the ellipse and lines, i.e., the projections of the model circle and the model lines, from image is an essential step to our new technique. Here we use random sample consensus paradigm (RANSAC) to extract lines and it proves performing well. In contrast to extracting lines, extracting the ellipse is a bit more involved. In our experiments reported in this paper, we use a least squares fitting technique based on algebraic distance to extract the ellipse. Assume that there are $n$ image points on the ellipse $\left\{\mathbf{x}_{i}\right\}=\left\{\left(x_{i}, y_{i}\right) i=1, \ldots, n\right\}$, and the equation of the ellipse is,

$$
Q(x, y)=A x^{2}+2 B x y+C y^{2}+2 D x+2 E y+F
$$

Then we minimize the cost function $\mathrm{F}=\sum_{i=1}^{n} Q^{2}\left(x_{i}, y_{i}\right)$ subject to the constraint of $A^{2}+B^{2}+C^{2}+D^{2}+E^{2}+F^{2}=1$ to extract the ellipse. More detailed discussions on extracting ellipses can be found in [8]. It is worth noting that generally speaking, using the Euclidean distance as the minimizing criterion will outperform that using algebraic distance in feature extraction. However, since our model plane is a white sheet containing a black circle and some black lines, its projected image is quite ideal, we found the extracted ellipse by minimizing the algebraic distance was already accurately enough, consequently we did not explore other more involved techniques.

\subsection{Determining the intrinsic parameters}

As discussed in section 2.1 and 2.2, once the image ( generally an ellipse) of the model circle and the vanishing line are obtained, the images of the two circular points $I_{m}$ 、 $J_{m}$ can be easily derived which are the intersecting points between the ellipse and the vanishing line.

Let $I_{m}=\left[I_{m 1}, I_{m 2}, I_{m 3}\right]^{T}$, and $C=K^{-T} K^{-1}=\left[\begin{array}{lll}C_{11} & C_{12} & C_{13} \\ C_{12} & C_{22} & C_{23} \\ C_{13} & C_{23} & C_{33}\end{array}\right]$

From (3), we have

$$
\left[I_{m 1}, I_{m 2}, I_{m 3}\right] C\left[I_{m 1}, I_{m 2}, I_{m 3}\right]^{T}=0
$$

Note that matrix $C$ is symmetric, we can define a $6 \times 1$ vector

$$
c=\left[C_{11}, C_{12}, C_{22}, C_{13}, C_{23}, C_{33}\right]^{T}
$$

Thus, (6) can be rewritten as

$$
A c=0
$$

With

$$
A=\left[I_{m 1} I_{m 1}, I_{m 1} I_{m 2}+I_{m 2} I_{m 1}, I_{m 2} I_{m 2}, I_{m 3} I_{m 1}+I_{m 1} I_{m 3}, I_{m 3} I_{m 2}+I_{m 2} I_{m 3}, I_{m 3} I_{m 3}\right]
$$

Since $A$ is a complex vector, $c$ is a real vector, (7) is equivalent to the following two homogeneous equations 


$$
\left[\begin{array}{c}
\operatorname{Re}(A) \\
\operatorname{Im}(A)
\end{array}\right] c=0
$$

If $n$ images of the model plane are observed, by stacking $n$ such equations, we have

$$
V c=0
$$

Where $V$ is a $2 n \times 6$ matrix. If $n \geq 3$ and $\operatorname{rank}(\mathrm{V}) \geq 5, c$ can be determined uniquely up to a scale factor in the least squares sense (If the skew factor $\gamma$ can be assumed to be zero in advance, then two images are sufficient for the calibration). The result can also be interpreted this way as shown in [9]: In general, 5 points are required to fit the image of the absolute conic, since each image can only provide two such points, the minimum number of images required is: $[5 / 2]=3$. The solution to (9) is well known as the eigenvector of $V^{T} V$ associated with the smallest eigenvalue [5].

Once vector $c$ is obtained, $K^{-1}$ can be computed using Cholesky factorization [5], and $K$ can then be obtained by inverting $K^{-1}$. This $K$ is equal to the actual camera intrinsic parameters matrix up to a scale. We can obtain the actual intrinsic parameters matrix by normalizing $K$ such that $k_{33}=1$.

\subsection{Recovering partial extrinsic parameters}

As indicated in section 2.1: Any point $(x, y, 0)$ in the model plane if satisfying $y= \pm i x$ ( $x 、 y$ are complex number) will correspond to $I_{m}$ (or $J_{m}$ ). Relating this to (2) we will have,

$$
s I_{m}=K\left[\begin{array}{lll}
\mathbf{r}_{1} & \mathbf{r}_{2} & \mathbf{t}
\end{array}\right]\left[\begin{array}{c}
x \\
\pm i x \\
0
\end{array}\right]=x\left(K \mathbf{r}_{1} \pm K \mathbf{r}_{2} \cdot i\right)
$$

Where $s$ is a constant, let $s / x=a+b i$, then

$$
\begin{aligned}
& \mathbf{r}_{1}=\lambda_{1} K^{-1}\left(a * \operatorname{Re}\left(I_{m}\right)-b * \operatorname{Im}\left(I_{m}\right)\right) \\
& \mathbf{r}_{2}=\lambda_{2} K^{-1}\left(b * \operatorname{Re}\left(I_{m}\right)+a * \operatorname{Im}\left(I_{m}\right)\right) \quad\left(\lambda_{1} 、 \lambda_{1} \text { are constants }\right)
\end{aligned}
$$

Where $a 、 b$ are unknown constants, so $\mathbf{r}_{1} 、 \mathbf{r}_{2}$ can not be uniquely obtained. It is worth noting that $\mathbf{r}_{1}^{T} \mathbf{r}_{2}=0$ is always true regardless of the takings of a and $b$, hence it cannot add any new more constraints on a and b. In the contrast, $\mathbf{r}_{3}$ can be derived from

Where $\lambda_{3}$ can be obtained by $\left\|\mathbf{r}_{3}\right\|=1$.

$$
\mathbf{r}_{3}=\lambda_{3}\left[K^{-1} \operatorname{Re}\left(I_{m}\right)\right] \times\left[K^{-1} \operatorname{Im}\left(I_{m}\right)\right]
$$

We can also recover $\mathbf{t}$ up to a unknown scale factor $\lambda_{t}$ by $m_{o}$, the image point of the model circle's center.

$$
\mathbf{t}=\lambda_{t} K^{-1} m_{O}
$$

Now we can see that the extrinsic parameters $\mathbf{r}_{1} 、 \mathbf{r}_{2}$ have been lost with our model plane. It is chiefly due to the centrosymmetry of our model plane. Suppose $C$ is the camera's optical center, and the origin of the camera coordinate system $X_{C} Y_{C} Z_{C}$ is

located at $C$ also. From section 2.1, we know that the coordinates of the circular points on the model plane are independent of the model circle's center $O$, thus without 
loss of generality, let $Z_{C}$ pass through the circle's center $O$ and let $O$ be the origin of the world coordinate system $X_{W} Y_{W} Z_{W}$, and $Z_{W}$ axis be perpendicular to the model plane. Under such a setup, vectors $\mathbf{r}_{1} 、 \mathbf{r}_{2} 、 \mathbf{r}_{3}$ of the rotation matrix $\mathrm{R}$ correspond to the unit vector of $X_{W} 、 Y_{W} 、 Z_{W}$ axes in the camera coordinate system, and the image of the model plane is only affected by $Z_{W}$ axis, but independent of $X_{W} 、 Y_{W}$ axes because of the centrosymmetry of the model plane. As a result, once camera's intrinsic parameters and the image of the model plane are obtained, we can only compute $\mathbf{r}_{3}$ from them.

Fortunately in most cases, the camera's extrinsic parameters are not required by DVS, so the loss of $\mathbf{r}_{1} 、 \mathbf{r}_{2}$ is not a major deficiency of our new technique.

\subsection{Degenerate configurations}

A complete analysis of all possible degenerate configurations in self-calibration has been carried out by Sturm [3] and Ma [7]. For our technique, degenerate configurations will only occur when the projected images of circular points in different images are identical. From (10) we can see that $I_{m}$ depends only on rotation matrix $R$ and is independent of translation vector t. Hence in order to avoid degenerate configurations, $R$ should be altered at each image taking. In other words, the relative orientation between the camera and the model plane must be different for different image taking.

Another degenerate configuration occurs when the image plane is parallel to the model plane. In this case, any point at infinity of the model plane $(x, y, 0,0)$ will correspond to

$$
K\left[\begin{array}{lll}
\mathbf{r}_{1} & \mathbf{r}_{2} & \mathbf{t}
\end{array}\right]\left[\begin{array}{l}
x \\
y \\
0
\end{array}\right]=K\left[\begin{array}{l}
x \\
y \\
0
\end{array}\right]=\left[\begin{array}{c}
\alpha x+\gamma y \\
\beta y \\
0
\end{array}\right]
$$

$[\alpha x+\gamma y, \beta y, 0]^{T}$ is a point at infinity of the image plane. It means that the vanishing line coincides with the line at infinity of the image plane. In this case, the method of computing the vanishing line in section 2.1 becomes invalid. Such a degenerate configuration can be easily detected and avoided as follows: assume $m_{O}$ is the image of the model circle's center, its corresponding polar line associated with the ellipse ( the projection of the model circle) is the vanishing line $l_{\infty}$. And we assume $E$ is the center of the ellipse whose corresponding polar line is the line at infinity of the image plane (denoted as $L_{\infty}$ ). According to the principle of polarity transformation in projective geometry, a polar line is uniquely determined by its corresponding polar associated with any proper quadric conic and vice versa. Hence, $l_{\infty}$ will coincide with $L_{\infty}$ as well as $m_{O}$ coincides with $E$ while image plane is parallel to the model plane. Based on this property, by checking the coincidence of $E$ and $m_{o}$, this degenerate configuration can be detected and avoided easily.

\subsection{Algorithm}

The proposed calibration algorithm can be outlined as follows:

1. Print a circle and a pencil of lines passing through the circle's center on a white sheet, and attach the sheet to a planar surface; 
2. Take 3 or more images of the sheet at different orientations;

3. For each image

3.1. Extract the ellipse and the pencil of lines, then check whether the configuration is a degenerate one as shown in section 2.6;

3.2. Compute the vanishing line as shown in section 2.2;

3.3. Compute the image points of the two circular points by intersecting the vanishing line with the ellipse;

4. Determine vector c and matrix $\mathrm{K}$ as shown in section 2.4 ,

\section{Experiments}

In this section, both synthetic and real experiments are reported.

\subsection{Simulation results}

In the computer simulations, the camera's setup is: $\alpha=1200, \beta=1000, \gamma=0.2$, $u_{0}=v_{0}=0$. The image resolution is: $1000 \times 1000$. The orientation of the model plane is characterized by a rotating axis $\mathrm{r}$ and a rotating angle $\theta$ about this axis (unit: degree). The position of the model plane is represented by a 3D vector $t$ (unit: centimeter). The model plane includes a circle with radius $=50$ (unit: centimeter) and 10 lines passing through the circle's center. The including angle between any two consecutive model lines is equal to 18 degrees. Standard deviations of the five intrinsic parameters at each different noise level are computed and shown in Figure 5. From this Figure, we can see that though the noise level increases to 6.0 pixels, the standard deviations of the intrinsic parameters are still low. This indicates that the proposed technique is accurate enough even with the presence of a high degree of noise.

\subsection{Experiments with real images}

Real images were taken with a KODAK-DC120 digital camera. The image resolution is $1280 \times 960$. We printed a circle with 6 lines passing through the circle's center on a white paper with a laser printer, and attached the paper to the wall. 4 images of the model plane were taken at different orientations as shown in Figure 4. Then we applied the proposed technique in this paper to these 4 images. The calibration results are shown in Table 1 .
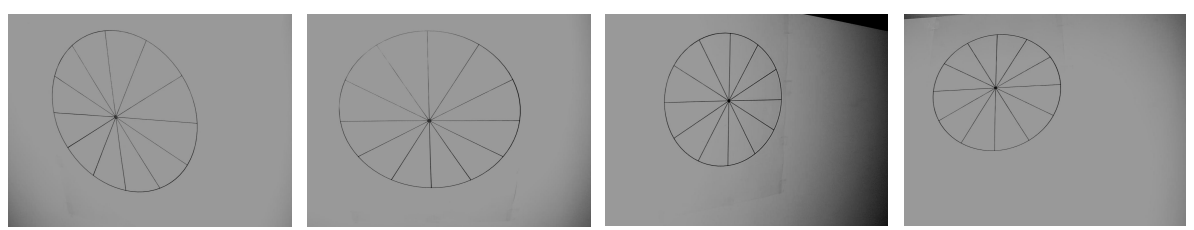

Figure 4: Four real images taken by digital camera 


\begin{tabular}{|c|c|c|c|c|c|}
\hline & $\alpha$ & $\beta$ & $\gamma$ & $\mathrm{u}_{0}$ & $\mathrm{v}_{0}$ \\
\hline Results & 1396.220 & 1400.731 & -0.827 & 568.401 & 453.079 \\
\hline
\end{tabular}

Table 1: Calibrating results from the 4 images in figure 4

In order to verify the truthfulness of the calibration results in Table 1, a wellstructured calibration pattern was reconstructed. Figure 6 consists of two images of the calibration pattern taken by previously calibrated camera. We manually picked 9 corresponding points from each one of the two visible sides (marked by cross). Applying the structure-from-motion algorithm as described in [4], we reconstructed the two visible sides. Two views of the reconstructed pattern under different view directions are shown in Figure 7. From Figure 7, we can see that reconstructed points on the same side of the calibration pattern are indeed coplanar. In addition, the computed including angle between the two reconstructed sides is $91.10^{\circ}$, which accords well with the ground truth (of $90.0^{\circ}$ ). This proves indirectly that the calibrating results in Table 1 are reliable.

\section{Conclusion}

Inspired by Zhang's technique, we proposed a new flexible calibrating technique based on the concept of circular points. Our new technique uses a novel model plane which includes a circle and a pencil of lines passing through the circle's center. The main points of our technique are: (1) It need not to establish point correspondences between the model points and resulting image ones, hence the calibration process can be fully automatic; (2) It need not to know the circle's center and radius, hence it is more convenient for the design and making of a model plane. The proposed technique is particularly useful for those people who are not familiar with computer vision. Experiments with simulated data as well as with real images show that our new technique is robust and accurate.

\section{References}

[1] Z.Zhang, Flexible camera calibration by viewing a plane from unknown orientations, Proc. ICCV'99, Vol.I: 666-673, 1999

[2] D.Liebowitz and A.Zisserman. Metric rectification for perspective images of planes. Proc. CVPR'98, 482-488, 1998.

[3] P.Sturm, Critical motion sequences for monocular self-calibration and uncalibrated Euclidean reconstruction, Proc. CVPR'97, 1100-1105, 1997.

[4] Z.Zhang, Motion and structure from two perspective views: From essential parameters to Euclidean motion via fundamental matrix, Journal of the Optical Society of America A, 14(11):2938-2950, 1997.

[5] G.Golub and C.van Loan, Matrix computations, The John Hopkins University Press, Baltimore, Maryland, third edition,1996

[6] J.G.Semple and G.T.Kneebone. Algebraic Projective Geometry. Oxford: Clarendon Press, 1952, Reprinted 1979. 
[7] Y. Ma, Stefano Soatto, Jana Kosecka, Shankar Sastry, Euclidean reconstruction and reprojection up to subgroups, Proc. ICCV'99, Vol. I: 773-780, 1999.

[8] Z.Zhang, Parameter estimation techniques: a tutorial with application to conic fitting, Technical Report, INRIA, October,1995

[9] O. Faugeras, L. Quan, and P. Sturm, Self-calibration of a 1D projective camera and its application to the self-calibration of a 2-D projective camera, Proc. ECCV'98, Vol.I, 36-54, 1998.

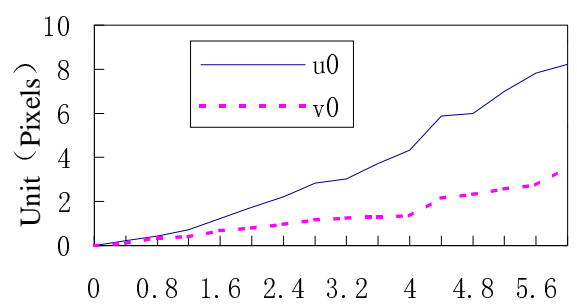

(a): Standard deviation of $\mathrm{u} 0, \mathrm{v} 0$

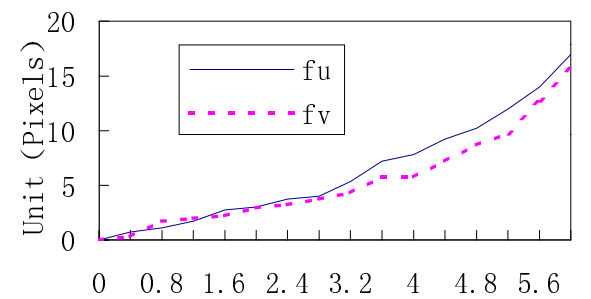

(b): Standard deviation of fu,fv

Figure 5: Calibrating results at different noise level
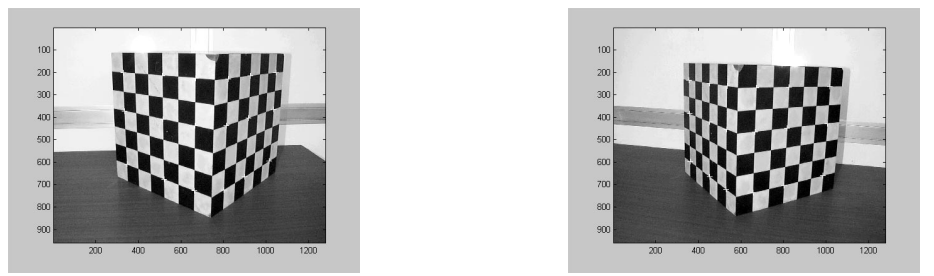

Figure 6: Two images of a calibration object taken by digital camera
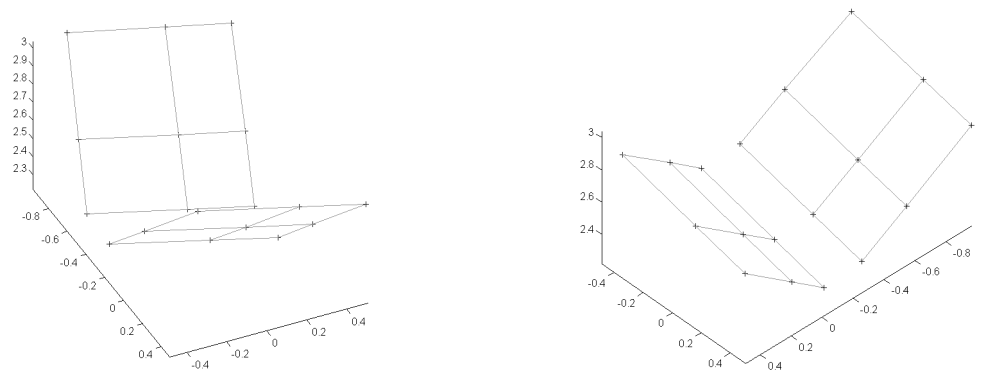

Figure 7: Two views of the reconstructed calibration object 